\title{
Evaluación de procesos de una intervención: actividad física durante el embarazo y postparto
}

\author{
Avaliação dos processos de uma intervenção: atividade física durante a gravidez \\ e o pós-parto
}

\section{Evaluation of intervention processes: physical activity during pregnancy and postpartum}

\author{
Ana Lilia Lozada-Tequeanes (iD \\ Instituto Nacional de Salud Pública (INSP) - Morelos - México
}

Lourdes Campero iD

Instituto Nacional de Salud Pública (INSP) - Morelos - México

Bernardo Hernández-Prado iD

University of Washington (UW) - Washington D.C. - Estados Unidos de América del Norte

Luis Rubalcava-Peñafiel iD

Spectron Desarrollo S.C. (SD) - Ciudad de México - México

\section{Lynnette Neufeld (iD}

Global Alliance for Improved Nutrition (GAIN) - Ginebra - Suiza

\section{RESUMEN}

Objetivo: Evaluar los procesos de una intervención de actividad física durante el embarazo y postparto. Métodos: En el contexto de un programa social que combate la pobreza extrema, las beneficiarias $(n=927)$ recibieron una intervención que consistió en la promoción de la práctica de actividad física, a través de consejería, talleres y materiales educativos. En 2008-9, 2010 y 2012 se visitaron unidades de salud urbanas y rurales, seleccionadas aleatoriamente, de cuatro entidades federativas de México. Se recolectó información de procesos a través de prestadores de servicios de salud, observación en consulta y aplicación de encuestas de salida a beneficiarias. Se estudiaron cuatro indicadores de implementación de la intervención: fidelidad a actividades planeadas; dosis entregada a la población; alcance de la población objetivo; y recepción de la intervención por embarazadas y en el posparto de las participantes. Resultados: Se visitaron unidades de salud en la etapa inicial $(n=91)$, intermedia $(n=47)$ y final $(n=82)$ del estudio. La dosis liberada presentó un nivel del $81-86 \%$ de implementación. La fidelidad presentó $<50 \%$ de implementación; se observaron mejoras significativas en la mayoría de los aspectos evaluados entre las diferentes etapas del estudio como en la capacitación de los prestadores de servicios de salud, el suministro de materiales, la consejería e la impartición de talleres en las unidades de salud. Conclusión: Se observaron importantes retos de implementación y contextuales para la implementación efectiva de la intervención de actividad física en los servicios de salud.

Descriptores: Evaluación de Proceso (Atención de Salud); Atención Primaria de Salud; Actividad Motora; Embarazo; Programas Sociales.

Comisiones de Ética en Investigación, Bioseguridad e Investigación del Instituto Nacional de Salud Pública de México (No. Cl768)

\section{RESUMO}

Objetivo: Avaliar os processos de uma intervenção de atividade física durante a gravidez e o pós-parto. Métodos: No contexto de um programa social de combate à pobreza extrema, as beneficiárias $(n=927)$ receberam uma intervenção que consistia na promoção da prática de atividade física por meio de aconselhamento, oficinas e materiais educacionais. Em 2008-9, 2010 e 2012, foram visitadas unidades de saúde urbanas e rurais, selecionadas aleatoriamente, de quatro entidades federais do México. As informações do processo foram coletadas por prestadores de serviços de saúde, durante observação de consultas e através da aplicação de questionários às beneficiárias. Foram estudados quatro indicadores de implementação da intervenção:

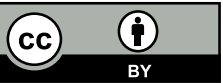


fidelidade às atividades planejadas, dose entregue à população, escopo da população-alvo, e acolhimento da intervenção por gestantes e puérperas. Resultados: As unidades de saúde foram visitadas nas etapas inicial $(n=91)$, intermediária $(n=47)$ e final ( $n=82)$ do estudo. A dose liberada mostrou um nível de implementação de $81-86 \%$. A fidelidade apresentou $<50 \%$ de implementação e melhorias significativas foram observadas na maioria dos aspectos avaliados entre as diferentes etapas do estudo, como treinamento de prestadores de serviços de saúde, fornecimento de materiais, aconselhamento e realização de oficinas nas unidades de saúde. Conclusão: Importantes implementações e desafios contextuais foram observados para a efetiva implementação da intervenção de atividade física para beneficiárias dos serviços de saúde investigados.

Descritores: Avaliação de Processos (Cuidados de Saúde); Atenção Primária à Saúde; Atividade Motora; Gravidez; Programas Sociais.

\section{ABSTRACT}

Objective: To evaluate the process of an intervention on physical activity during pregnancy and postpartum. Methods: In the context of a social program that fights extreme poverty, the beneficiaries $(n=927)$ receive an intervention that consists of the promotion of the practice of physical activity, through counseling, workshops, and educational materials. During 2008-9, 2010 and 2012, we visited health units from urban and rural areas, randomly selected from four states of Mexico. Health service providers collected process data during observation of consultations and through the application of questionnaires to the beneficiaries. Four indicators of implementation of the intervention were studied: fidelity to the planned activities, dose delivered to the population, scope of the target population, and reception of the intervention by pregnant women and postpartum women. Results: Health units were visited in the initial $(n=91)$, intermediate $(n=47)$, and final $(n=82)$ stages of the study. The delivered dose showed an implementation level of $81-86 \%$. Fidelity had $<50 \%$ implementation and significant improvements were observed in most analyzed aspects between the different stages of the study, such as training of health service providers, the supply of materials, counseling, and workshops in health units. Conclusion: Important Implementation and contextual challenges were observed for the effective fulfillment of the intervention of physical activity into primary healthcare services.

Descriptors: Process Assessment (Health Care); Primary Health Care; Motor Activity; Pregnancy; Social Programs.

\section{INTRODUCCIÓN}

La promoción de la salud se concibe como la suma de acciones que la población, los servicios, las autoridades sanitarias y otros sectores sociales y productivos desarrollan a fin de mejorar las condiciones de salud y la calidad de vida de las personas y sus comunidades. Asimismo, busca involucrar a las personas para incrementar su autocontrol en pro de bienestar ${ }^{(1)}$.

En este sentido, en años recientes, los organismos internacionales han emitido recomendaciones sobre los beneficios y la relevancia del desarrollo e implementación de políticas y programas que promuevan la práctica regular de actividad física (PAF) a lo largo del ciclo vital, en particular, como medida preventiva de enfermedades crónicas ${ }^{(1)}$. De manera específica, la PAF ha presentado diversas barreras individuales y socio-culturales para su desarrollo durante el embarazo y periodo posparto (embarazo-pp). Entre las principales limitaciones están los prejuicios de género $^{(2)}$, la falta de apoyo social para las mujeres y la falta de información o consejería durante la consulta pre y posnatal por parte de los prestadores de servicios de salud (PSS) ${ }^{(2)}$.

Entre las pocas intervenciones existentes que incorporan la PAF durante el embarazo y posparto ${ }^{(3)}$, la mayoría se encuentra en países desarrollados. No obstante, su implementación y evaluación, nos muestra lecciones valiosas en torno a sus estrategias educativas efectivas o deficientes, formas para contactar con las participantes ${ }^{(4)}$ y ventajas de la capacitación a los PSS para incrementar conocimientos ${ }^{(5)}$ a favor de su recomendación entre las mujeres que atienden.

Si bien, en las últimas décadas la evaluación de procesos y programas han ido en aumento pero la evidencia aún es escasa en escenarios de países de medianos y bajos ingresos ${ }^{(6)}$. En México, el Programa Prospera (antes Oportunidades) otorgaba a familias que viven en extrema pobreza, el beneficio de atención en la salud, y en particular, a mujeres embarazadas o en lactancia ${ }^{(7)}$.

En 2008-9, el Prospera desarrolló e implementó la Estrategia Integral de Atención a la Nutrición (EsIAN). Ésta buscó promover el cambio en conductas en múltiples temas ${ }^{(8,9)}$, y se conformó de tres componentes: a) Campaña de comunicación efectiva para promover mensajes claves en relación a la nutrición y $\operatorname{PAF}^{(10)}$; b) Capacitación y sensibilización del PSS sobre temas de interés y uso de comunicación efectiva; y c) Equipamiento e insumos en las unidades de salud (US). 
Este artículo se centra en la EsIAN para beneficiarias con embarazo-pp, con el objetivo de evaluar los procesos de una intervención de actividad física durante el embarazo y el postparto ${ }^{(11,12)}$.

\section{MÉTODOS}

En México, el Programa Prospera, a través de Estrategia Integral de Atención a la Nutrición (EsIAN), incluye una intervención de actividad física (IAF) en embarazo-pp, que además cuenta con diferentes mediciones en el tiempo, lo que permite documentar los diferentes niveles de implementación de un programa de salud poblacional.

Entre 2008 y 2012, el Programa Prospera implementó la EsIAN como un estudio de factibilidad en población beneficiaria para cuatro entidades federativas de la República Mexicana (Estado de México, Guerrero, Michoacán y Puebla). La selección de dichas entidades se basó en su alta densidad de beneficiarios y en su región de ubicación dentro del país (Centro, Occidente y Sur). Se incluyeron localidades urbanas (>15,000 habs.) y rurales (no indígenas y predominantemente indígenas) que tenían prevalencia alta (>20\%) o moderada (10-20\%) de talla baja en menores de 5 años de edad. La unidad de intervención fue la US (unidades de salud), a partir de una selección aleatoria considerando los recursos monetarios disponibles. La EsIAN contó con medición basal en 140 US (2008-9), un seguimiento intermedio de 47 US (2010) y una etapa final de 91 US (2012). En esta última etapa se conformó la muestra poblacional de 927 participantes con las beneficiarias en embarazo-pp que vivían en la zona de cobertura de la US y que estaban expuestas a EsIAN.

A partir de un diagnóstico previo al estudio para conocer a mayor detalle a la población objetivo (investigación formativa) se desarrolló, como parte del primer componente de EsIAN (comunicación efectiva), un único mensaje clave introductorio a estilos de vida saludable para intervención de actividad física (IAF) en embarazo-pp. Este se perfeccionó y validó siguiendo la metodología de mercadeo social(13) cuidando que fuera culturalmente aceptable para la población de interés: "Camina entre 20 y 30 minutos cada día a paso rápido, así mejoras tu salud y la de tu bebé. Invita a tu familia, es más divertido y es bueno para todos". La promoción del cambio en conducta se apoyó con imágenes que enmarcaban diferentes contextos de la vida cotidiana y de recreación.

Para el componente de EsIAN (capacitación y sensibilización de PSS), se diseñó un programa en cascada con niveles y pasos secuenciados. El primer nivel estuvo a cargo del equipo de investigación el cual constó de una sesión intensiva con responsables estatales, quienes trasmitirían la información a los jefes jurisdiccionales de salud y éstos a su vez, bajarían la información entre PSS en funciones dentro de la US (segundo nivel). Como resultado final, se esperaba se proporcionar la información y consejería a la beneficiaria a través de los diferentes contactos que se tuviera con las mujeres tanto en la consulta pre y posnatal, como en talleres educativos en salud (tercer nivel). Para la capacitación en cascada se utilizaron siempre los mismos recursos educativos (videos, ejercicios, evaluaciones, entre otros); además de los mismos contenidos: sensibilización, reforzamiento y homogeneización de conocimientos en relación a los temas de interés (en este caso sobre PAF en embarazo-pp) y orientación sobre comunicación efectiva.

En cuanto al componente tres (equipamiento y suministros en la US), Prospera proporcionó a las US suplementos nutricionales (ej: tabletas de micronutrimentos para el embarazo y posparto), fotómetros portátiles, equipo antropométrico, así como, los materiales educativos referentes a los temas de interés de EsIAN (ej: lactancia materna, alimentación complementaria, anemia, sobrepeso/obesidad, talla baja, alimentación saludable y actividad física, incluyendo lo específico de la PAF en embarazo-pp). Los materiales educativos que se distribuyeron fueron manuales técnicos y rotafolios ilustrados que promovían las diferentes conductas deseadas en la población objetivo. Para esto y a fin de llegar a la población con baja escolaridad, se diseñaron imágenes sencillas a color y mensajes cortos específicos para la promoción de cada conducta ${ }^{(3)}$.

En la Tabla I se muestra la ruta esperada de la IAF, que lleva a la mejora de la salud de la beneficiaria.

Se consideraron cuatro indicadores para la evaluación de procesos (Cuadro I): 1) Dosis liberada: Porcentaje de actividades de la IAF entregadas a la población objetivo por PS respecto al total esperado (ej. consejería en consulta o impartición del taller de salud). 2) Fidelidad: Proporción de componentes de IAF completadas en la US que se adhieren a lo planeado; aproximación de la calidad de la implementación (ej. identificación del mensaje clave y uso de material EsIAN por parte del PS). 3) Dosis recibida: Proporción de beneficiarias que recuerdan la exposición a las actividades de intervención (ej. reconocen mensaje o imagen clave sobre PAF en embarazo-pp). 4) Alcance: Porcentaje de la población objetivo que participa en la IAF.

También, en la etapa final, se contó con información sobre el contexto de entrega (ej. edad, lengua indígena, lugar de residencia, semanas de embarazo, etc.) de la IAF entre beneficiarias. 
Para la recolección de datos se siguió el siguiente procedimiento. Un equipo de encuestadores, estandarizados y capacitados, recabó en 1.5 días de estancia en la localidad de estudio, información de PSS y beneficiarias presentes en el día y medio de trabajo. Al llegar a la US, se explicaba y solicitaba autorización para llevar a cabo la recolección de información; y se cuidó no avisar con anticipación dicha visita, para evitar la modificación de la atención habitual. Con la finalidad de disminuir los sesgos de respuesta, se evitó hacer aclaraciones sobre PAF. La encuesta consistió en plantear la pregunta, sin mencionar o sugerir opciones de respuesta; por lo que, la información se colectó bajo la interpretación que cada participante comprendió(14). Los momentos en el tiempo, las variables generadas a partir de los reactivos de las encuestas y tamaños de muestra de los indicadores se presentan en el Cuadro I.

Para el análisis de datos, se siguió el siguiente proceso. Una doble captura y la limpieza de las bases de datos, se estimaron medias y proporciones crudas de las variables de interés. Para estimar las prevalencias ajustadas se utilizaron modelos de regresión logística y comandos de post-estimación (adjust/margins). Se consideraron variables de ajuste con base en la revisión de la literatura y por suficiencia de observaciones para la estimación del modelo. Los estimadores consideraron el diseño de muestreo por conglomerados (US en este caso). La significancia estadística fue declarada para un valor $p<0.05$.

Para la interpretación de los datos se consideró como mínimo aceptable $90 \%$ de la capacitación del PSS y $90 \%$ del suministro de materiales educativos ${ }^{(15)}$. La consejería en consulta fue considerada aceptable con el $80 \%{ }^{(16)}$ y la impartición de talleres en salud con el $50 \%$. Por último, en el caso de fidelidad, dosis liberada, dosis recibida y alcance, como promedio de las variables de implementación fueron referidas como modestas $\geq 50 \%$ y como adecuadas con $>90 \%$. Todos los análisis estadísticos fueron realizados en el Stata v14 ${ }^{(17)}$.

Cabe mencionar que el protocolo para la evaluación de la EsIAN fue aprobado por las Comisiones de Ética en Investigación, Bioseguridad e Investigación del Instituto Nacional de Salud Pública de México (No. Cl768). Todos los participantes dieron su consentimiento oral y por escrito.

Tabla I - Modelo lógico de la intervención sobre practica de actividad física en el embarazo y posparto, EsIAN. Programa Prospera. México, 2008-9/2012.

\begin{tabular}{|c|c|c|c|c|}
\hline Insumos & $\begin{array}{l}\text { Resultados esperados } \\
\text { inmediatos }\end{array}$ & $\begin{array}{c}\text { Resultados esperados } \\
\text { de corto plazo }\end{array}$ & $\begin{array}{c}\text { Resultados } \\
\text { esperados de } \\
\text { mediado a largo } \\
\text { plazo }\end{array}$ & Efectos en salud \\
\hline $\begin{array}{l}\text { - Proveer capacitación } \\
\text { a proveedores de salud } \\
\text { - Suministrar materiales } \\
\text { educativos y equipo } \\
\text { esencial en la Unidad } \\
\text { de salud (US) }\end{array}$ & $\begin{array}{l}\text { - Aumentar conocimientos y } \\
\text { capacidades respecto a la } \\
\text { PAF en el embarazo y PP en } \\
\text { proveedores de salud }\end{array}$ & $\begin{array}{l}\text { - Consejería en consulta } \\
\text { sobre PAF en el embarazo } \\
\text { y PP } \\
\text { - Impartición de talleres } \\
\text { sobre PAF en el embarazo } \\
\text { y PP } \\
\text { - Uso de materiales } \\
\text { educativos sobre PAF en } \\
\text { embarazo y lactancia } \\
\text { - Reconocimiento de } \\
\text { mensajes claves e imágenes } \\
\text { de mujeres embarazadas } \\
\text { o en PP usuarias de los } \\
\text { servicios de salud }\end{array}$ & $\begin{array}{l}\text { - Aumento de PAF en } \\
\text { el embarazo y PP por } \\
\text { mujeres beneficiarias del } \\
\text { programa Oportunidades }\end{array}$ & $\begin{array}{l}\text { - Ganancia apropiada } \\
\text { de peso gestacional } \\
\text { - Menor retención de } \\
\text { peso posparto } \\
\text { - Aumento en salud } \\
\text { de las mujeres } \\
\text { beneficiarias de } \\
\text { PDHO }\end{array}$ \\
\hline
\end{tabular}

PAF: práctica de actividad física; PP: posparto; PDHO: Programa de Desarrollo Humano Oportunidades

\section{RESULTADOS}

A continuación, se presentan los principales resultados para cada uno de los indicadores considerados en la evaluación de procesos e implementación de la IAF.

La dosis liberada de IAF no se completó adecuadamente para ninguna de los seguimientos del estudio de EsIAN (Tabla II). La capacitación del PSS no presentó cambios significativos ya que pasó del $81 \%$ en 2010 a $86 \%$ en 2012 $(p>0.05)$. Se observó una falta importante en la entrega de materiales educativos en 2010 y en 2012 ( $p>0.05)$. La 


\begin{tabular}{|c|c|c|c|c|c|c|c|c|}
\hline 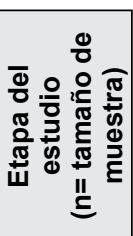 & 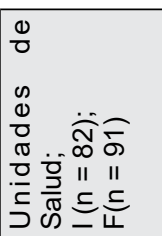 & 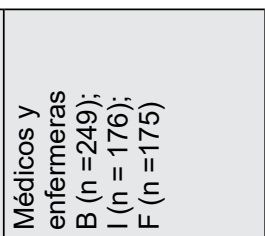 & 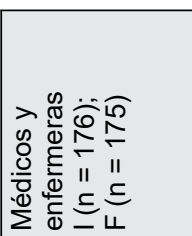 & 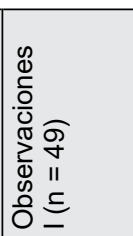 & 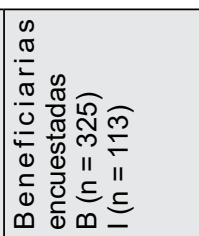 & 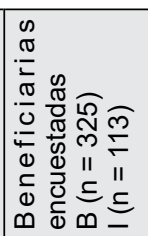 & 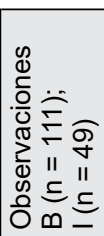 & 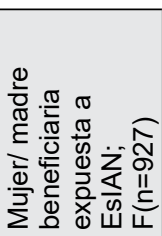 \\
\hline 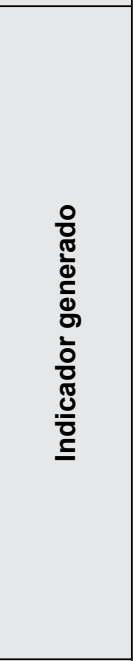 & 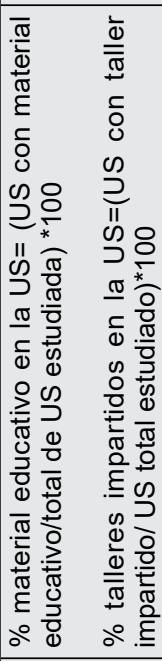 & 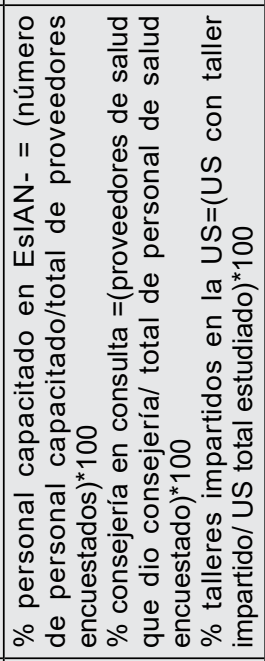 & 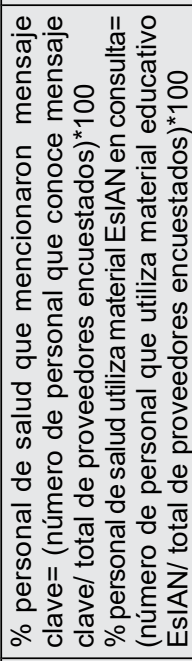 & 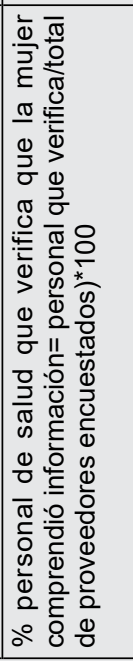 & 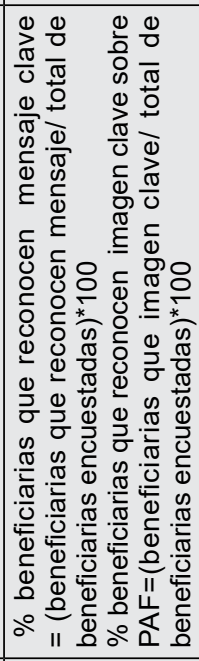 & 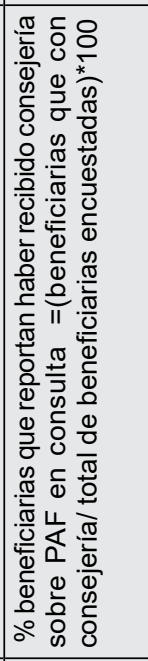 & 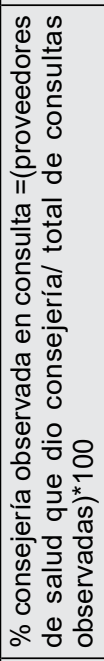 & 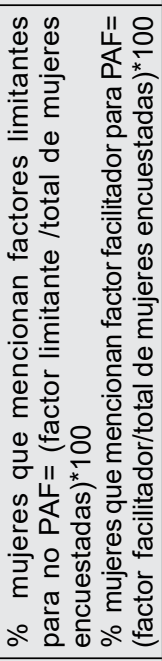 \\
\hline 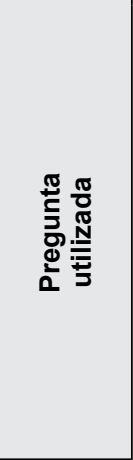 & 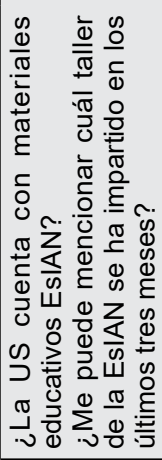 & 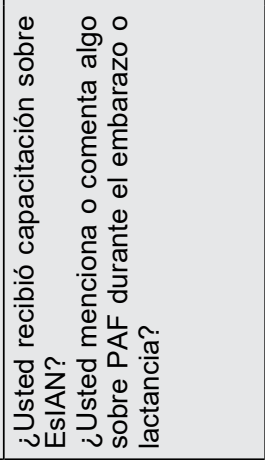 & 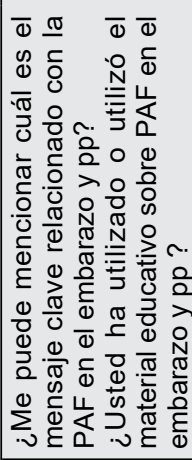 & 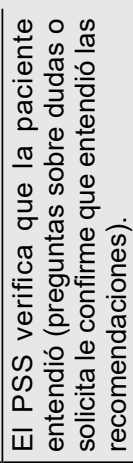 & 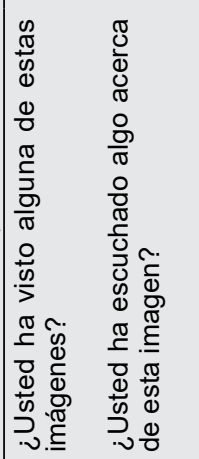 & 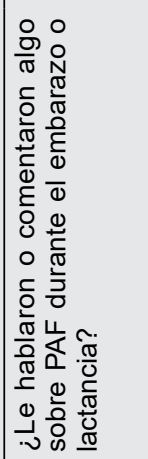 & 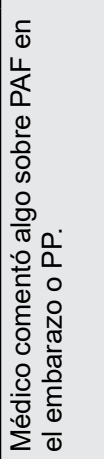 & 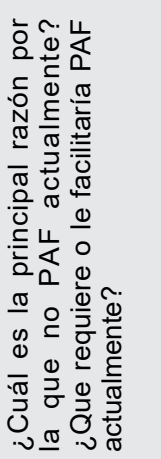 \\
\hline 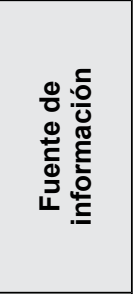 & 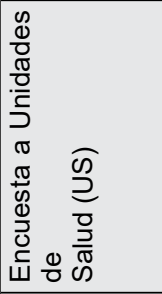 & 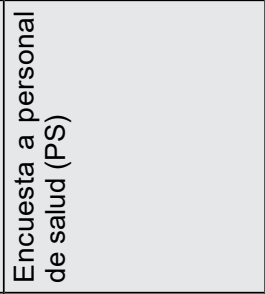 & 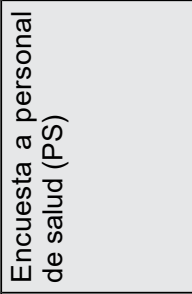 & 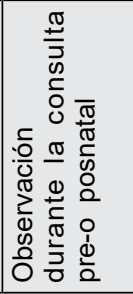 & 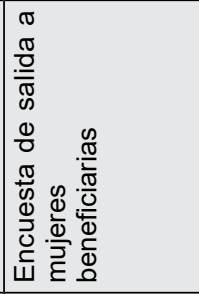 & 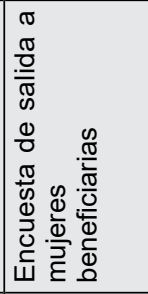 & 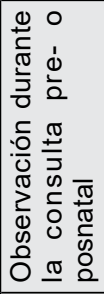 & 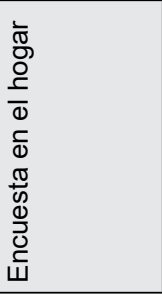 \\
\hline 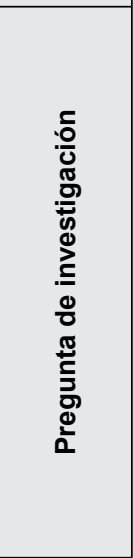 & 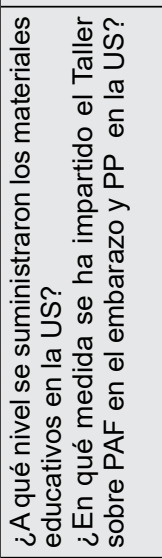 & 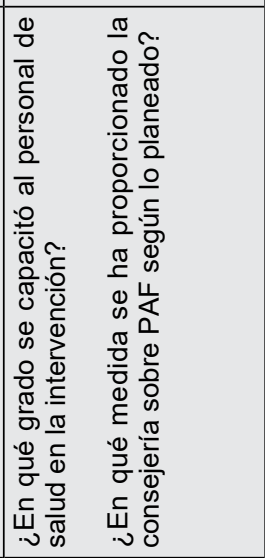 & 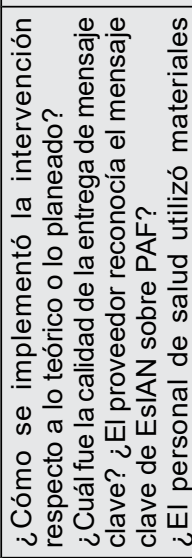 & 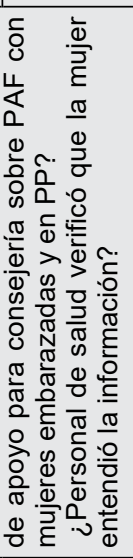 & 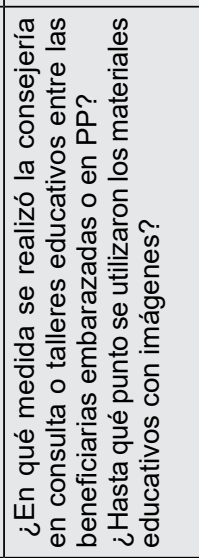 & 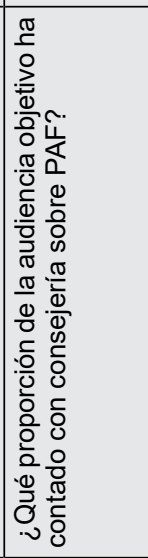 & 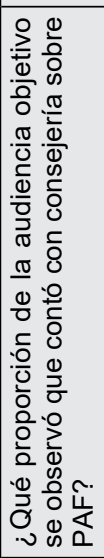 & 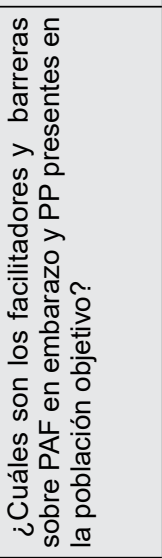 \\
\hline 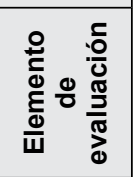 & & 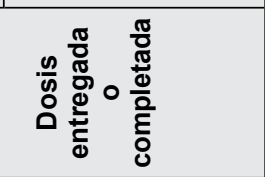 & $\begin{array}{l}\frac{\pi}{0} \\
\frac{\pi}{0} \\
\frac{0}{0} \\
\frac{0}{4}\end{array}$ & & 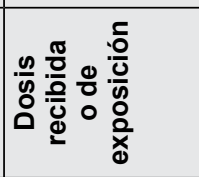 & 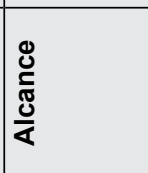 & & 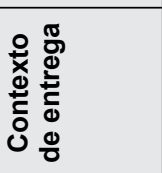 \\
\hline
\end{tabular}


consejería sobre PAF en consulta pre y posnatal, se registró desde la medición basal, previo a la intervención, y presentó un incremento entre los seguimientos $(p<0.05)$. Por otra parte, la impartición de talleres educativos en la US presentó la proporción más baja en la implementación. Cabe mencionar, que sólo la consejería en consulta de la impartición de talleres alcanzó el mínimo aceptable.

Por otro lado, la fidelidad de la implementación de IAF con la que se llevaron a cabo las actividades de IAF fue categorizada como modesta $(<50 \%)$. No obstante, se observó que el reconocimiento del mensaje clave sobre PAF por PS presentó un aumento significativo entre los seguimientos (del $18.6 \%$ a $87.5 \%$; $p<0.05$ ).

Como dosis recibida de la IAF por las beneficiarias, se manejó como reconocimiento y recuerdo espontaneo de la imagen y mensaje clave asociado con PAF en embarazo-pp, por parte de la beneficiaria, presentó proporciones de 30 y $40 \%$, respectivamente (Tabla I). Al comparar la etapa inicial vs intermedia, se identificó un alcance de casi el doble (del $38 \%$ a $73 \%$; $p<0.05$ ) entre las beneficiarias que refirieron haber recibido consejería sobre PAF en la consulta. De forma similar, la observación directa en consulta documentó el aumento de consejería sobre PAF entre etapas de evaluación de EsIAN (24 a 41\%; p<0.05).

Tabla II - Prevalencias ajustadas de los elementos de evaluación para cada componente de la intervención de actividad física (IAF) en embarazo y posparto en diferentes etapas de implementación de EsIAN, Programa Prospera*. México, 2008-9/2012.

\begin{tabular}{|c|c|c|c|}
\hline \multirow[b]{2}{*}{ Componentes de la intervención } & \multicolumn{3}{|c|}{ Etapas y año de la etapa del estudio } \\
\hline & $\begin{array}{c}\text { Basal } \\
2008-9 \\
\text { (n) }\end{array}$ & $\begin{array}{l}\text { Intermedia } \\
2010 \\
\text { (n) }\end{array}$ & $\begin{array}{l}\text { Final } \pi \\
2012 \\
\text { (n) }\end{array}$ \\
\hline \multicolumn{4}{|l|}{ Dosis completada (\%) } \\
\hline Personal de salud recibió capacitación de la EsIAN & 0 & $\begin{array}{c}81.0 \\
(n=132)\end{array}$ & $\begin{array}{c}85.7 \\
(n=161)\end{array}$ \\
\hline Existencia de materiales educativos EsIAN en la unidad de salud & 0 & $\begin{array}{c}60.7^{b} \\
(n=107)\end{array}$ & $\begin{array}{c}86.2 \\
(n=87)\end{array}$ \\
\hline Consejería otorgado por personal de salud sobre PAF en consulta & $\begin{array}{c}57.7 \\
(n=45)\end{array}$ & $\begin{array}{l}55.1^{\mathrm{b}} \\
(\mathrm{n}=58)\end{array}$ & $\begin{array}{c}87.5^{c} \\
(n=152)\end{array}$ \\
\hline Talleres sobre PAF impartidos por personal de salud en la unidad & 0 & $\begin{array}{l}32.9^{b} \\
(n=88)\end{array}$ & $\begin{array}{c}59.2 \\
(n=54)\end{array}$ \\
\hline \multicolumn{4}{|l|}{ Fidelidad (\%) } \\
\hline $\begin{array}{l}\text { Identificación por personal de salud del mensaje clave sobre PAF } \\
\text { de EsIAN }\end{array}$ & 0 & $\begin{array}{c}18.6^{b} \\
(n=107)\end{array}$ & $\begin{array}{c}87.5 \\
(n=153)\end{array}$ \\
\hline $\begin{array}{l}\text { Uso de materiales EsIAN observado por personal de la evaluación } \\
\text { durante consulta }\end{array}$ & 0 & $\begin{array}{c}27.5 \\
(n=29)\end{array}$ & -- \\
\hline $\begin{array}{l}\text { Observación que el proveedor confirma que la mujer entendió la } \\
\text { información }\end{array}$ & 0 & $\begin{array}{c}82.3 \\
(n=34)\end{array}$ & -- \\
\hline \multicolumn{4}{|l|}{ Dosis recibida $(\%)$} \\
\hline Mujer beneficiaria reconoció imagen de PAF en el embarazo & 0 & $\begin{array}{c}30.0 \\
(n=30)\end{array}$ & -- \\
\hline $\begin{array}{l}\text { Mujer beneficiaria recordó o enunció espontáneamente mensaje } \\
\text { clave sobre PAF }\end{array}$ & 0 & $\begin{array}{c}40.0 \\
(n=20)\end{array}$ & -- \\
\hline \multicolumn{4}{|l|}{ Alcance (\%) } \\
\hline Mujer beneficiaria refirió haber recibido consejería sobre PAF & $\begin{array}{l}37.9^{a} \\
(n=45)\end{array}$ & $\begin{array}{c}73.0 \\
(n=26)\end{array}$ & -- \\
\hline Personal de evaluación observó consejería sobre PAF durante consulta & $\begin{array}{c}24.4^{a} \\
(n=90)\end{array}$ & $\begin{array}{c}41.1 \\
(n=34)\end{array}$ & -- \\
\hline
\end{tabular}

"Las preguntas planteadas en EsIAN final difieren de etapas previas; las diferencias en $\mathrm{n}$ respecto a la cuadro 1, se deben a la perdida de observaciones, debido a los missing de las variables utilizadas en el ajuste o por etapa del estudio. *Las proporciones fueron calculadas por individuo (personal de salud o mujer beneficiaria), consulta observada o US

*Ajuste por muestreo por conglomerados. Proporciones ajustadas según: Encuesta de personal de salud se ajustó por edad, sexo y puesto; Encuesta a mujer beneficiaria se ajustó por edad y área de residencia; Encuesta a la Unidad de Salud se ajustó por dependencia de salud que la opera (SSA o IMSS) y área de residencia); formato de observación se ajustó por edad del

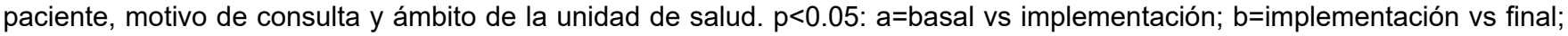
$\mathrm{c}=$ basal vs final

PAF: práctica de actividad física; PP: posparto; EsIAN: Estrategia Integral de Atención a la Nutrición 
Finalmente, se observa que la implementación se completó a diferentes niveles, y sólo el alcance fue modesto $(>50 \%)$ y la dosis liberada (>90\%) adecuada (Figura 1$)$.

Por otro lado, es importante decir que entre los factores contextuales, se detectó como principal barrera para $\mathrm{PAF}$, no tener quien cuide a sus hijos(as) y/o atienda al esposo. Entre los requerimientos para integrar la PAF como conducta cotidiana, se documentó el apoyo en el cuidado de los hijos(as) (26.3\%) y tener los aditamentos necesarios, tales como: dinero, ropa, etc. $(11 \%)(p<0.05)$. (Tabla III).

Se resalta que solamente el $10 \%$ de las beneficiarias expresaron que no les gusta la PAF, sin diferencia entre zonas urbanas y rurales; no obstante, indicaron disposición para realizarla.

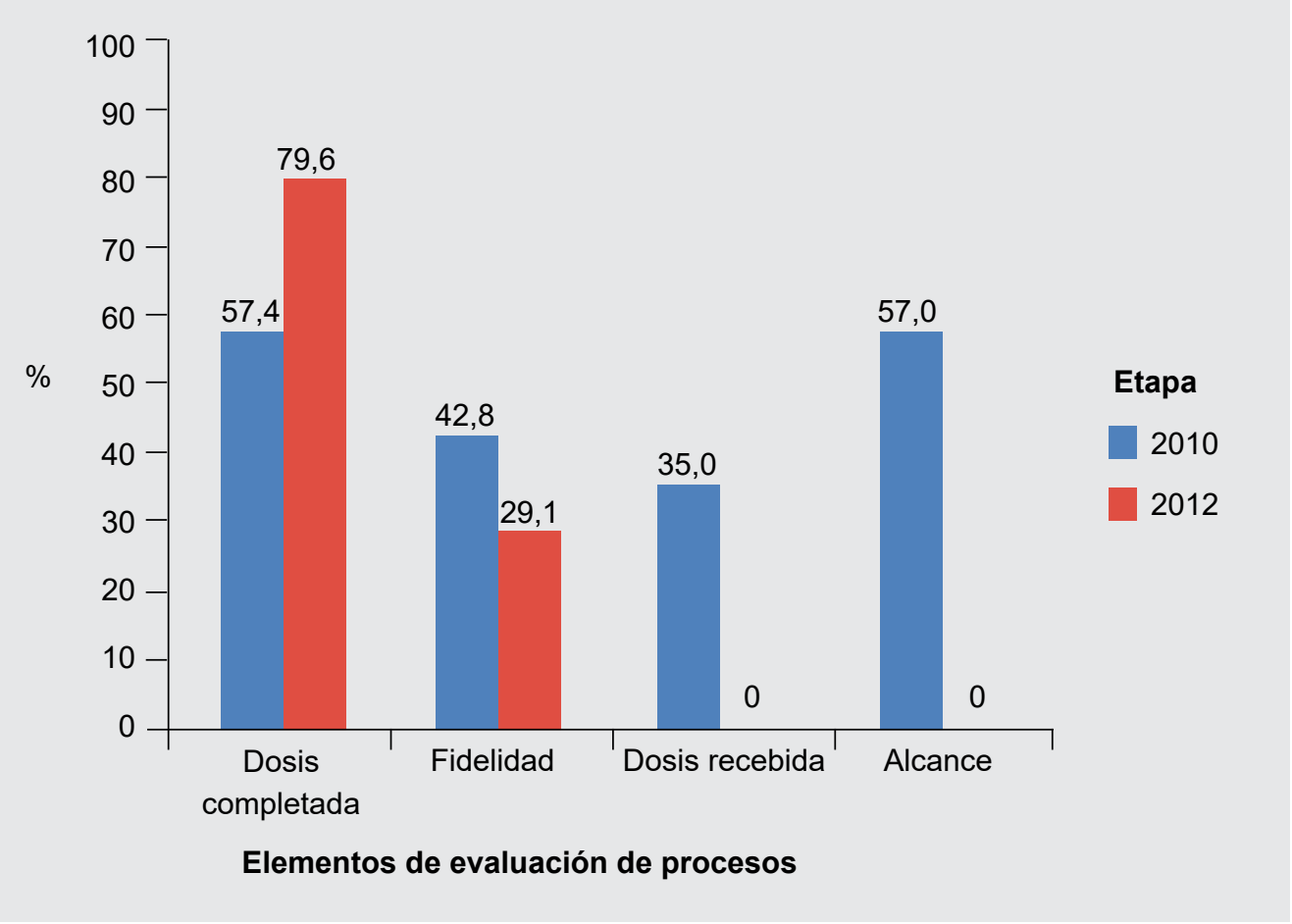

Figura 1 - Elementos de proceso en diferentes etapas de evaluación de una intervención educativa que promueve la PAF en embarazo y posparto. EsIAN, Programa Prospera*.

"Las preguntas planteadas en EsIAN final difieren de etapas previas; Las proporciones representan el promedio de la suma de los porcentajes de las variables que componen cada elemento de evaluación, así mismo, las preguntas que componen cada variable se presentan en la Tabla I. Los datos de 2008-9 no se presentan, dado que, en esa etapa el nivel de implementación de fidelidad, dosis y alcance sería cero, y es hasta la etapa intermedia que fue posible estimar proporciones de los elementos de procesos de la intervención

PAF: práctica de actividad física; PP: posparto; EsIAN: Estrategia Integral de Atención a la Nutrición

Tabla III - Variables relacionadas con la práctica de actividad física durante el embarazo y posparto de beneficiarias del Programa Prospera de la zona rural y urbana. EsIAN, 2012. (n=927)*. México, 2008-9/2012.

\begin{tabular}{lcc}
\hline Variables & $\begin{array}{c}\text { Rural (n=370) } \\
\%(n) \text { ó } X \pm D E\end{array}$ & $\begin{array}{c}\text { Urbano (n=551) } \\
\%(n) \text { ó } X \pm D E\end{array}$ \\
\hline Edad (años) $^{£}$ & $32.2 \pm 7.60$ & $30.8 \pm 7.33$ \\
Embarazo actual & 1.3 & 2.7 \\
& $(12)$ & $(25)$ \\
Actualmente practica actividad & 30.1 \\
Entre las que practican, número de veces por semana & $(n=156)$ & $(n=281)$ \\
Entre las que practican, el tipo de actividad física más común & $3.9 \pm 2.3$ & $4.0 \pm 1.99$ \\
$\quad$ Caminar & & \\
$\quad$ Correr & $20.9(91)$ & $31.4(137)$ \\
$\quad$ Baile & $2.7(12)$ & $8.5(37)$ \\
Otro (bicicleta, aerobics, etc.) & $5.0(22)$ & $12.8(56)$ \\
\hline
\end{tabular}




\begin{tabular}{lcc}
\hline Entre las que no practican, principal razón & $(\mathrm{n}=214)$ & $(\mathrm{n}=278)$ \\
No lo recomienda personal de salud & $1.4(7)$ & $1.2(6)$ \\
No le gusta & $10.3(51)$ & $10.3(51)$ \\
No es seguro & $0.6(3)$ & $0.6(3)$ \\
No tiene quien cuide a sus hijos/esposo & $13.1(65)$ & $23.7(117)$ \\
No hay infraestructura física & $1.8(9)$ & $0.8(4)$ \\
No hay instructor & $0.4(2)$ & $0.2(1)$ \\
Otro & $15.0(74)$ & $19.0(94)$ \\
Entre las que no practican, disposición de practicarla en algún momento & $51.8(\mathrm{n}=255)$ \\
Entre las que no practican pero están dispuestas, requieren para realizar & $36.9(\mathrm{n}=182)$ & \\
actividad físicaf & & $1.6(7)$ \\
Que lo recomiende el personal de salud & & $4.8(21)$ \\
Que le guste o disfrute & $0.4(2)$ & $17.7(77)$ \\
Que alguien cuide a sus hijos & $3.6(16)$ & $1.1(5)$ \\
Tener compañía para PAF & $8.5(37)$ & $3.6(16)$ \\
Infraestructura física & $1.3(6)$ & $1.8(8)$ \\
Que haya instructor & $3.4(15)$ & $3.4(15)$ \\
Dinero, ropa, tenis, etc. & $3.6(16)$ & $21.6(94)$ \\
Otro & $6.6(29)$ & $10.3(46)$ \\
\hline
\end{tabular}

EsIAN: Estrategia Integral de Atención a la Nutrición; PP: posparto. *Ajuste por muestreo

\section{DISCUSIÓN}

Este trabajo documenta la evaluación de procesos de una IAF realizada en el primer nivel de atención en salud con mujeres en embarazo-pp incorporadas a Prospera en cuatro estados de la república mexicana. Esta evaluación de procesos involucra el uso de indicadores que reflejan cómo la intervención se entregó y recibió por porte de la población participante en esta investigación ${ }^{(18)}$.

El presente estudio mostró incremento en la consejería en consulta sobre PAF (dosis entregada) y beneficiarias que refirieron haber recibido consejería y la consejería observada en consulta (alcance), lo cual sugiere que, el conocimiento que el PSS obtuvo en la capacitación de EsIAN fue utilizado en su práctica cotidiana. No obstante, se ha documentado que el cambio de comportamiento en PSS requiere una infraestructura que facilite su trabajo, así como, de capacitación ${ }^{(6)}$. En este sentido, Prospera cuenta con una estructura física y de organización bien establecida, lo que facilita e influye en prácticas de atención en el PSS. Por otra parte, la IAF fue pensada para ser incorporada dentro de las actividades cotidianas en la misma consulta que se ofrece a la mujer, ya que se sabe que tener fuertes presiones de tiempo ${ }^{(19)}$. En análisis secundarios ${ }^{(20)}$ referentes a esta IAF, se detectó que un factor que pudo influir en la incorporación de la consejería sobre PAF por el PSS fue su edad, ya que estos eran relativamente jóvenes (media $=35.6 \pm 9.3$ años) y por lo mismo, pudiera ser que los hace más susceptibles a aceptar ideas nuevas de manejo médico en su práctica cotidiana. Por otro lado, un alcance no adecuado pudo deberse a la rotación excesiva del PSS (ej. cambios de $17 \%$ en la plantilla del PS entre seguimientos) ${ }^{(6)}$.

Por otro lado, aunque la retención de información a corto plazo, supone el primer pasó en el proceso de cambio de comportamiento, se observó que solo una proporción baja de beneficiarias recordaron la conducta sobre PAF al momento de salir de la US (dosis recibida). Lo anterior, puede deberse a que, aunque los mensajes fueron diseñados en un "lenguaje común", la familiarización por PSS requirió énfasis en la capacitación a fin de haberse transmitido a la beneficiaria. Se ha documentado la mayor parte del tiempo de una consulta se dedica a regaños y llamadas de atención y saturación de información ${ }^{(15,21)}$, sin que el paciente se quede con elementos prácticos que pueda llevar a su realidad. Ante esto, la capacitación hacia PSS debe incluir técnicas que minimicen dicho escenario para optimizar el tiempo y mensajes, así como resolver "barreras" de la población objetivo.

Una implementación modesta se ha documentado por otros autores ${ }^{(22)}$, no obstante, ese nivel ha mostrado efectos positivos $^{(23)}$. En Balagar, Nueva Delhi, la intensidad de la intervención para prevenir enfermedades crónicas creció hasta alcanzar un nivel crítico con efectos significativos, cuando su nivel de implementación se mantuvo. Sin embargo, también se ha señalado que un interés constante por parte de los tomadores de decisiones, es indispensable ${ }^{(24)}$. 
Un estudio en India que compara los resultados en dos regiones, señala que mientras en una región hubo un incremento de PAF como resultado de un programa focalizado, en contraste con la otra que presentó inactividad física por ser inapropiado para las mujeres ejercitarse ${ }^{(25)}$. Esta experiencia, muestra que si bien las intervenciones pueden ser modificadas o adaptadas según el contexto de entrega, los factores socio-culturales podrían limitar la IAF entre beneficiarias, por lo que es importante considerar focalización y tiempo para medir efectos a gran escala.

Existe escasa evidencia sobre la evaluación de procesos de intervenciones para promover PAF durante embarazopp. Entre los estudios que documentan indicadores de proceso, se identifican pocos ${ }^{(26,27)}$, no obstante, generalmente estos no se detienen a explicar detalladamente el proceso que se siguió para su diseño e implementación de su IAF, y directamente se van a reportar resultados. Entre las fortalezas de este trabajo, está el de utilizar múltiples fuentes de información y metodología mixta ${ }^{(28)}$ ya que incrementa la probabilidad de alcanzar los objetivos planteados ${ }^{(29)}$. A la vez, en este estudio se cuenta con la información tanto de participantes como de implementadores de la $\operatorname{IAF}^{(30)}$ y se hace un análisis detallado para evaluar indicadores de procesos en diferentes momentos en el tiempo.

Como limitaciones de este estudio, podrían mencionarse algunas. Como era de esperarse, la recolección de información por observación generó reactividad inherente en PSS y en beneficiarias; dicha reactividad puede ser difícil de estimar y no se puede descartar su efecto en lo observado( ${ }^{(31)}$. No obstante, no se espera que esta influencia se haya modificado entre etapas del estudio, por lo que, el aumento significativo en consejería podría ser resultado de la capacitación y no de la reactividad. A pesar del compromiso de las instituciones a cargo de proveer los suministros para EsIAN, éstos sufrieron atrasos importantes por lo que, se debe trabajar cercanamente con los tomadores de decisiones para tratar de aminorar situaciones que afecten negativamente la implementación de las intervenciones. Por otro lado, los datos muestran una inminente necesidad de reforzar la capacitación en cascada del PSS. Una opción podría ser la capacitación a distancia que reduce costos, tiempo e incrementa la cobertura.

Por último, cabe resaltar la poca importancia que se da en los programas a la evaluación de procesos y de impacto, lo que propició la falta de apoyo político-administrativo y restricciones financieras para colectar información completa en todas las etapas. Lo que a la vez limitó la disponibilidad de información para la realización de análisis estadísticos más robustos y que se pudieran estimar incluso inferencias causales.

La experiencia aquí descrita tiene como fin último documentar la evaluación de la implementación de programas y permite explicar las posibles rutas de acción, documentarlas y retroalimentar la implantación de otras intervenciones poblacionales. Si no se evalúan los procesos, se asume que la implementación es adecuada a lo planeado y se corre el riesgo de no tener ningún impacto. Lo anterior, es relevante dado el escenario de recursos limitados y problemas de salud pública prevalentes ${ }^{(32)}$, y en donde, se requieren intervenciones factibles y sostenibles que permitan la promoción de la salud en las poblaciones.

\section{CONCLUSIÓN}

Se observaron importantes retos de implementación y contextuales para la implementación efectiva de la intervención de actividad física en los servicios de salud.

Cabe recordar que Estrategia Integral de Atención a la Nutrición (EsIAN) se implementó bajo condiciones habituales de operación en las unidades de salud, y no como un estudio controlado de investigación. Por lo anterior, los resultados son altamente extrapolables a una amplia diversidad de contextos.

\section{CONFLICTO DE INTERÉS}

Los autores y autoras de este trabajo declaran no tener conflicto de interés.

\section{FUENTES DE FINANCIAMIENTO}

Beca de Doctorado del Consejo Nacional de Ciencia y Tecnología (CONACYT).

\section{CONTRIBUCIONES}

Ana Lilia Lozada-Tequeanes y Lourdes Campero contribuido a la preparación y diseño del estudio; la adquisición, análisis e interpretación de datos; y la redacción y / o revisión del manuscrito. Bernardo Hernández-Prado y Luis Rubalcava-Peñafiel contribuido a la adquisición, análisis e interpretación de datos; y la redacción y / o revisión del manuscrito. 


\section{REFERÊNCIAS}

1. World Health Organization. Global Action Plan on Physical Activity 2018-2030: more Active People for a Healthier World. Geneva: WHO; 2018.

2. Lozada AL, Campero L, Hernández B, Rubalcava L, Neufeld LM. Múltiples factores individuales, comunitarios y ambientales limitan la práctica de actividad física durante el embarazo y posparto en mujeres pobres de México. Salud Pública Mex. 2015;57:242-51.

3. International Weight Management in Pregnancy (i-WIP) Collaborative Group. Effect of diet and physical activity based interventions in pregnancy on gestational weight gain and pregnancy outcomes: meta-analysis of individual participant data from randomized trials. BMJ. 2017;358:j3119.

4. Peters D, Tran N, Adam T. Implementation research in health: a practical guide. Geneva: WHO; 2013.

5. González W, Bonvecchio Arenas A, García-Guerra A, Vilar-Compte M, Villa de la Vega A, Quezada L, et al. An iterative process for training design and implementation increased health workers knowledge for tacking nutrition behavior change to scale. J Nutr. 2019;149(S1):2323S-31S.

6. Robert RC, Gittelsohn J, Creed-Kanashiro HM, Penny ME, Caulfield LE, Narro MR, et al. Process evaluation determines the pathway of success for a health center-delivered, nutrition education intervention for infants in Trujillo, Peru. J Nutr. 2006;136:634-41.

7. Secretaría de Bienestar (MEX). Reglas de Operación del Programa Prospera (antes Programa de Desarrollo Humano Oportunidades) [Internet]. México: Gobierno de México; 2019. [Acesso em 2020 Jan 29]. Disponível em: https://www.gob.mx/cms/uploads/attachment/file/442955/Reglas_de_Operacion_PROSPERA_2019.pdf

8. Tougas ME, Hayden JA, McGrath PJ, Huguet A, Rozario S. A systematic review exploring the social cognitive theory of self- regulation as a framework for chronic health condition interventions. PLoS ONE. 2015;10(8):119.

9. Maibach EW, Rothschild ML, Novelli WD. Social marketing. In: Glanz K, Lewis RM, Rimer BK, editores. Health behavior and health education: theory, research and practice. San Francisco, CA: Jossey-Bass; 2002. p. 437-61.

10. Shuval K, DiPietro L, Skinner CS, Barlow CE, Morrow J, Goldsteen R, et al. Sedentary behavior counselling: the next step in lifestyle counselling in primary care; pilot findings from the Rapid Assessment Dissue Index (RADI) study. Br J Sports Med. 2014;48(19):1451-5.

11. Menon P, Covic MN, Harrigan BP, Horton ES, Kazi MN, Lamstein S, et al. Strengthening implementation and utilization of nutrition interventions through research: a framework and research agenda. Ann N Y Acad Sci. 2014;1332:39-59.

12. Tumilowicz A, Ruel MT, Pelto G, Pelletier D, Monterrosa E, Lapping K, et al. Implementation Science in Nutrition: Concepts and Frameworks for an Emerging Field of Science and Practice. Curr Dev Nutr. 2018;3(3):nzy080.

13. French J. Key concepts, principles and techniques of social marketing. In: Social marketing and public health: theory and practice. New York, NY: Oxford University Press; 2017. p. 17-25.

14. Hernández-Ávila M, editor. Epidemiología. Diseño y análisis de estudios. Cuernavaca, MEX: Instituto Nacional de Salud Pública; 2015.

15. Bonvecchio Arenas A, González W, Théodore FL, Lozada-Tequeanes AL, Garcia-Guerra A, Alvarado R, et al. Translating evidence-based program recommendations into action. The design, testing and scaling up of the behavior change strategy EsIAN in Mexico. J Nutr. 2019;149:2310S-22S.

16. Robert RC, Creed-Kanashiro HM, Villasante R, Narro MR, Penny ME. Strenghtening health services to deliver nutrition education to promote complementary feeding and healthy growth of infants and young children: formative research for a successful intervention in peri-urban Trujillo, Peru. Matern Child Nutr. 2017;13(2):115.

17. StataCorp. Stata Statistical Software: release 12. College Station, TX: StataCorp LP; 2018. 
18. Consejo Nacional de Evaluación. Modelo de términos de referencia de Evaluación de Procesos [Internet]. Ciudad de México: CONEVAL; 2017 [consultado 10 Jan 2016]. Disponible en: https://www.coneval.org.mx/ Evaluacion/MDE/Documents/TDR_Procesos.pdf

19. Prasad K, Poplau S, Brown R, Yale S, Grossman E, Varkey AB, et al. Time pressure during primary care office visits: a prospective evaluation of data from the healthy work place study. J Gen Inter Med. 2019.

20. Neufeld LM. Diseño y fortalecimiento programático basado en evidencia: el caso de nutrición en el programa Progresa-Oportunidades-Prospera. In: Hernández LG, De la Garza TZ, Janet-Yaschine I. EI ProgresaOportunidades-Prospera: a 20 años de su creación [Internet]. Ciudad de México: CONEVAL; [fecha desconocida]. Disponible en: https://www.coneval.org.mx/Evaluacion/IEPSM/Documents/Libro_POP_20.pdf

21. Delisle H, Shrimpton R, Blaney S, Du Plessis L, Atwook S, Sanders D, et al. Capacity-building for a strong public health nutrition workforce in low-resource countries. Bull World Health Organ. 2017;95:385-8.

22. Shojania KG. Conventional evaluations of improvement interventions: more trials or just more tribulations? BMJ Qual Saf. 2013;22(11):881-4.

23. Dickey VC, Pachón H, Marsh DR, Lang TT, Claussenius DR, Dearden KA, et al. Implementation of nutrition education and rehabilitation programs (NERPs) in Viet Nam. Food Nutr Bull. 2002;23(4 Suppl):78-85.

24. Neufeld LM, Grados R, Villa de la Vega A, Steta C, Regalia F, Rivera-Dommarco JA. A brief history of evidence-informed decision making for nutrition in Mexico. J Nutr. 2019;149:2277S-80S.

25. Mohan V, Shanthirani CS, Deepa M, Datta M. Community empowerment: a successful model for prevention of non-communicable diseases in India: the Chennai Urban Population Study (CUPS -17). J Assoc Phys India. 2006;54:858-62.

26. Paul $\mathrm{KH}$, Olson $\mathrm{CM}$. Moving beyond quantity of participation in process evaluation of an intervention to prevent excessive pregnancy weight gain. Int J of Behavioral Nutr and Phys Act. 2013;10:8-11.

27. Kinnunen TI, Aittasalo M, Koponen P, Ojala K, Mansikkamaki K, Weiderpass E, et al. Feasibility of a controlled trial aiming to prevent excessive pregnancy-related weight gain in primary health care. BMC Pregnancy Childbirth. 2008;8:37.

28. Poblete CM. Métodos mixtos: una aproximación a sus ventajas y limitaciones en la investigación de sistemas y servicios de salud. Rev Chil Salud Pública. 2013;17(3):218-23.

29. Östlund U, Kidd L, Wengstro"m Y, Rowa-Dewar N. Combining qualitative and quantitative research within mixed method research designs: a methodological review. Int J Nurs Stud. 2011;48:369-83.

30. Gillespie S, Menon P, Kennedy AL. Scaling up impact on nutrition: what will it take? Adv Nutr. 2015;6(4):44051.

31. Schnelle JF, Ouslander JG, Simmons SF. Direct observations of nursing home care quality: Does care change when observed? J Am Med Dir Assoc. 2006;7(9):541-4.

32. Sharma S, Adetoro OO, Vidler M, Drebit S, Payne BA, Akeju DO, et al. A process evaluation plan for assessing a complex community-based maternal health intervention in Ogun State, Nigeria. BMC Health Serv Res. 2017;17(1):238.

\author{
Dirección para correspondencia: \\ Lourdes Campero \\ Instituto Nacional de Salud Pública (INSP) \\ Av. Universidad 655 \\ Col. Santa María Ahuacatitlán \\ CP. 62100 - Cuernavaca - Morelos - México \\ E-mail: Icampero@insp.mx
}


Dirección del primer autor:

Ana Lilia Lozada-Tequeanes

Instituto Nacional de Salud Pública (INSP)

Av. Universidad 655

Col. Santa María Ahuacatitlán

CP. 62100 - Cuernavaca - Morelos - México

E-mail: alilote@gmail.com

Como citar: Lozada-Tequeanes AL, Campero L, Hernández-Prado B, Rubalcava-Peñafiel L, Neufeld L. Evaluación de procesos de una intervención: actividad física durante el embarazo y postparto. Rev Bras Promoç Saúde. 2020;33:9635. 